\title{
Transposition
}

Musique et Sciences Sociales

Musique : patrimoine immatériel ?

\section{La notion de patrimoine immatériel comme outil de contournement de l'État : enjeux et ambiguïés de la fabrique patrimoniale du gwoka en Guadeloupe}

The Notion of ICH as a Means of Circumventing the State:

Issues and Ambiguities of Gwoka's Heritagisation Process in Guadeloupe

Florabelle Spielmann et Dominique Cyrille

\section{(2) OpenEdition}

Journals

Édition électronique

URL : http://journals.openedition.org/transposition/3468

DOI : 10.4000/transposition.3468

ISSN : 2110-6134

Éditeur

CRAL - Centre de recherche sur les arts et le langage

\section{Référence électronique}

Florabelle Spielmann et Dominique Cyrille, « La notion de patrimoine immatériel comme outil de contournement de l'État : enjeux et ambiguïtés de la fabrique patrimoniale du gwoka en Guadeloupe », Transposition [En ligne], 8 | 2019, mis en ligne le 15 septembre 2019, consulté le 16 décembre 2020. URL : http://journals.openedition.org/transposition/3468; DOI : https://doi.org/10.4000/transposition. 3468

Ce document a été généré automatiquement le 16 décembre 2020.

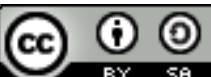

La revue Transposition est mise à disposition selon les termes de la Licence Creative Commons Attribution - Partage dans les Mêmes Conditions 4.0 International. 


\section{La notion de patrimoine immatériel comme outil de contournement de l'État : enjeux et ambiguïtés de la fabrique patrimoniale du gwoka en Guadeloupe}

The Notion of ICH as a Means of Circumventing the State:

Issues and Ambiguities of Gwoka's Heritagisation Process in Guadeloupe

Florabelle Spielmann et Dominique Cyrille

\section{Introduction}

1 Paris, le 26 novembre 2014: le gwoka de Guadeloupe est inscrit sur la Liste Représentative (LR) du Patrimoine Culturel Immatériel (PCI) de l'humanité de l'UNESCO. La Guadeloupe, un ensemble de sept îles et îlots situés au milieu de l'archipel Caraïbe, fait partie de ces terres des Amériques où, à partir de 1640, la France réduit en esclavage des milliers d'humains kidnappés en Afrique de l'Ouest. Quand en 1848 l'esclavage est enfin aboli, la culture amérindienne originelle a laissé la place à de nouvelles expressions culturelles où dominent les influences africaines. Le gwoka est né de celles-ci. Musique de tambour et de chant responsorial, le gwoka traditionnel se décline aujourd'hui en sept rythmes caractéristiques exprimés simultanément par des tambours appelés ka et des danseurs solistes. Il se joue la nuit, lors de soirées festives appelées léwoz.

2 En 1946, la Guadeloupe entre dans la nation française en tant que département d'outremer, fait unique au regard du processus de décolonisation observé ailleurs. La départementalisation ne tenant pas ses promesses, les années 1960 voient l'émergence d'un mouvement séparatiste qui construit son discours politique sur une base 
culturelle identitaire ${ }^{1}$ et place le gwoka au cœur de son action. Jusque-là qualifié de mizik a vyé nèg (musique des gens déconsidérés ${ }^{2}$ ), le gwoka devient un symbole identitaire et un objet de fierté pour une majorité de Guadeloupéens. Cependant, son inscription sur la LR du PCI a suscité des tensions qui ont ravivé « un débat récurrent sur le thème du lien séculaire les unissant à la France ${ }^{3} »$.

Cet article souhaite contribuer à la réflexion sur les logiques d'appropriation du concept de patrimoine culturel. Il examine l'impact du processus d'inscription du gwoka sur la LR du PCI de l'UNESCO et met en lumière les enjeux politiques de cette appropriation pour les acteurs du gwoka. Il ne s'intéresse pas au gwoka en tant qu'objet d'étude - il ne se penche ni sur son histoire, ni sur son esthétique. Il interroge plutôt les spécificités du gwoka dans ses interactions avec le paradigme du Patrimoine Culturel Immatériel : que fait le "patrimoine immatériel » au gwoka ? Et inversement, que fait le gwoka au "patrimoine immatériel »? Cet article est en partie basé sur des données ethnographiques recueillies entre 2006 et 2015 par les auteures dans le cadre d'une observation participante.

De septembre 2008 à novembre 2015, Dominique Cyrille a été responsable de la mission Patrimoine du Centre des musiques et danses traditionnelles et populaires de Guadeloupe (Centre Rèpriz), dont le président était Félix Cotellon. Avocat, militant culturel, Félix Cotellon a œuvré pour la reconnaissance du gwoka en Guadeloupe. Il est notamment à l'origine de la création du Festival de Gwoka de Sainte-Anne à la fin des années 1980. Tirant les enseignements du processus d'inscription du maloya à La Réunion, où un débat virulent a eu lieu a posteriori ${ }^{4}$, Félix Cotellon a souhaité qu'en Guadeloupe la population soit pleinement informée afin qu'elle puisse porter en amont un avis éclairé sur ce projet d'inscription. Dans la continuité de sa participation à des journées de réflexion organisées par la Maison des Cultures du Monde et par la Fédération des Associations de Musique et Danse Traditionnelles (FAMDT), Félix Cotellon a souhaité que des chercheurs soient associés aux projets de sauvegarde du patrimoine guadeloupéen et donc aussi au projet d'inscription du gwoka. A cette fin, Dominique Cyrille a été sollicitée pour créer et diriger la mission Patrimoine du Centre des musiques et danses traditionnelles et populaires de Guadeloupe. Dans le cadre de ses fonctions, elle a contribué à la mise en place et à la coordination d'une trentaine de personnes impliquées dans le milieu du gwoka (musiciens, danseurs, chanteurs, chercheurs, responsables d'association, avocats...). Appelé "Lyannaj pou Gwoka», ce groupe d'acteurs du gwoka a porté le projet d'inscription. De son côté, Florabelle Spielmann a travaillé à la Maison des Cultures du Monde à Paris entre mai 2007 et novembre 2009 sous la direction de Chérif Khaznadar puis d'Arwad Esber. Entre 2010 et 2014, elle a été membre du comité « Lyannaj pou Gwoka » en Guadeloupe à la demande de Dominique Cyrille. À ce titre, Florabelle Spielmann a travaillé avec des musiciens et des chanteurs à l'élaboration du texte d'identification du gwoka, aujourd'hui mis en ligne sur le site de l'UNESCO. Dominique Cyrille a facilité les réunions relatives aux mesures de sauvegarde. Ensemble, elles ont participé à la préparation du dossier de candidature du gwoka à la Liste Représentative du PCI.

5 Par ailleurs, Félix Cotellon, attentif aux critères de recevabilité du dossier d'inscription, a souhaité que des actions de communication et de consultation du public soient mises en place à plusieurs niveaux. Dominique Cyrille et Florabelle Spielmann ont accompagné la mise en œuvre de ces actions : appel au soutien du projet d'inscription présenté et déposé dans toutes les mairies de Guadeloupe, rédaction d'une brochure de 
présentation du projet remise aux instances politiques (Conseil régional, Conseil général, mairies), rédaction d'une brochure d'état d'avancement à mi-parcours destinée au grand public (plus de mille exemplaires), rédaction de contenus et communication avec la presse et les médias par internet, facilitation des réunions de travail hebdomadaires du «Lyannaj pou Gwoka » sur une période d'environ huit mois (quatre réunions par semaine), présentation du projet d'inscription au public dans le cadre de réunions et de manifestations publiques, participation aux débats publics sur le projet d'inscription organisés par des associations culturelles, etc.. Ainsi, Félix Cotellon a trouvé une façon originale de mettre en application les Directives Opérationnelles de la Convention en associant des chercheurs à la conception et à la mise en œuvre d'un éventail d'actions qui ont favorisé la co-construction par les acteurs du gwoka et les chercheurs d'un processus d'inscription adapté au contexte guadeloupéen. Dans la continuité des questions soulevées par Guillaume Samson ${ }^{5}$ et par Christian Hottin ${ }^{6}$, l'exemple de la Guadeloupe est un cas d'étude qui contribue à la réflexion en cours sur la place du chercheur dans les processus de mise en patrimoine.

Cet article commence par une présentation du Rapport culturel publié par l'Association Générale des Étudiants Guadeloupéens (AGEG) en 1970. Ce Rapport culturel est un texte fondateur qui développe « une revendication spécifique privilégiant le gwoka comme élément central de la personnalité culturelle populaire guadeloupéenne ${ }^{7} »$. Ce document est à l'origine des premiers efforts de mise en patrimoine du gwoka par les Guadeloupéens eux-mêmes dans les années 1970 et 1980. Nous nous intéresserons ensuite à la mise en circulation de la notion de $\mathrm{PCI}$ en Guadeloupe. Contrairement à ce qui a pu être observé ailleurs, cette mise en circulation a pour particularité d'avoir été initiée par les porteurs de tradition ${ }^{8}$ et non par l'État. Enfin nous nous arrêterons sur les débats passionnés que le projet d'inscription a suscités, transformant le gwoka en « arène patrimoniale ${ }^{9}$.

\section{Le Rapport culturel : un manifeste politique}

7 Au lendemain de la guerre, la Guadeloupe devient un département d'outre-mer français par la loi de départementalisation du 19 mars 1946. La fin du statut institutionnel de colonie prend ainsi la forme d'une intégration formelle à la nation française. Mais rapidement, face à la persistance des inégalités sociales et des clivages nés de la subordination coloniale, l'organisation jacobine de l'État français et son modèle assimilationniste ${ }^{10} d^{\prime}$ 'intégration suscitent des prises de position critiques à l'encontre du pouvoir. Dans un contexte mondial de décolonisation, dans un contexte local de désillusions, un activisme politique guadeloupéen s'organise en réaction au modèle social et culturel porté par l'État français. Perçue comme une «forme suprême de colonialisme $\mathrm{e}^{11}$ » et vécue comme une forme d'oppression, l'assimilation culturelle est contestée et combattue dans le cadre de projets politiques émancipateurs prenant d'abord forme au sein de l'Association Générale des Étudiants Guadeloupéens (AGEG). Créée en 1928 à Bordeaux, cette association avait initialement pour mission de tisser des liens de solidarité entre les étudiants guadeloupéens, de défendre leurs intérêts, et de faciliter leur insertion professionnelle en France hexagonale. Après la départementalisation (1946), ce groupe associatif investit le terrain politique en se positionnant contre l'assimilation culturelle en 1960, en se prononçant pour l'indépendance nationale de la Guadeloupe en 1963, et en créant un journal 
indépendantiste, Le patriote guadeloupéen, «au service de la lutte du peuple guadeloupéen ».

En 1970, à l'issue du $9^{\mathrm{e}}$ congrès de l'AGEG, les membres de l'association rédigent un document de soixante-dix pages intitulé Rapport culturel. Ce texte propose une approche radicalement nouvelle, centrée sur la «lente maturation» qui a accompagné la conception $\mathrm{du}$ projet politique indépendantiste, nationaliste, anticolonialiste et anticapitaliste du camp patriotique guadeloupéen. Y sont exposées les modalités d'action de l'AGEG. Cette association propose de construire un projet politique indépendantiste en s'appuyant sur une " expérience culturelle » et sur " un effort de recherche et d'analyse ${ }^{12}$ » effectué entre 1958 et 1968, combinant études historiques et observation directe de la société guadeloupéenne. C'est en mettant en œuvre ce projet politique que se tissent des liens entre travailleurs agricoles et « camarades étudiants » qui s'investissent dans les luttes sociales de la classe ouvrière et paysanne en participant notamment à la création des premiers syndicats représentant les «masses laborieuses ${ }^{13}$ » : l'Union des Travailleurs Agricoles et l'Union des Paysans Pauvres de la Guadeloupe. Les difficultés économiques continuent de stimuler la formation de nouveaux syndicats jusqu'à la création en 1978 de l'Union Générale des Travailleurs Guadeloupéens (UGTG). Ces syndicats, qui organisent des manifestations culturelles et dispensent des cours d'éducation populaire en soirée dans les campagnes, favorisent une mise en relation des intellectuels avec les larges masses populaires et permettent «de connaître un peu mieux le peuple, ses aspirations, ses besoins, sa vie ${ }^{14}$ ». Se positionnant contre le parti communiste ${ }^{15}$, considéré comme le «soutien le plus actif $\mathrm{du}$ colonialisme en Guadeloupe », ces militants politiques diffusent des idées révolutionnaires pour organiser les travailleurs et ouvrir la voie à une «Lutte de Libération Nationale qui [doit] aboutir à l'Indépendance Nationale de la Guadeloupe ${ }^{16}$ ".

En s'interrogeant sur les fondements de la domination économique, idéologique et culturelle propre à la société guadeloupéenne, les rédacteurs de ce rapport insistent sur la dimension culturelle de l'exercice du pouvoir. Ainsi, la contestation de l'ordre social et des infrastructures économiques des années 1960/70 (grèves d'ouvriers, de travailleurs du bâtiment, de personnels hôteliers...) doit s'accompagner d'un «front culturel » ainsi présenté dans l'introduction de ce document :

Dans la grande lutte qu'il a déclenchée pour son indépendance et sa liberté, le peuple guadeloupéen est engagé sur tous les fronts. Le front de la culture est un de ceux-là. Il y a plus de trois siècles, le colonialisme français commença à exploiter notre pays. À mesure que son oppression s'affermissait, il instituait tout un système politique, militaire, culturel chargé de maintenir et de renforcer la base économique colonialiste. Les institutions culturelles, l'art et la littérature furent pour lui des moyens au service de ces objectifs. Face à cette agression, le peuple guadeloupéen a entrepris de résister et de vaincre. Il a entrepris d'utiliser toutes les armes de la culture pour combattre l'aliénation, la départementalisation et de développer le sentiment naturel guadeloupéen ${ }^{17}$.

10 Se référant à Lénine et à Staline, la conception du pouvoir énoncée dans ce Rapport culturel renvoie aux théories politiques développées par Antonio Gramsci et plus particulièrement à son concept d'hégémonie culturelle. Les rédacteurs du rapport affirment que l'hégémonie de la bourgeoisie guadeloupéenne se serait construite à partir du concept d'assimilation culturelle défendue par l'État français dans le cadre de la loi de départementalisation. Selon eux, cette assimilation culturelle a permis de 
perpétuer la soumission de la population guadeloupéenne à la domination coloniale devenue domination capitaliste.

Dénonçant les effets de ce processus d'assimilation culturelle ayant garanti l'hégémonie d'une classe bourgeoise "qui s'est faite l'alliée zélée des propagandistes colonialistes ${ }^{18}$ ", les rédacteurs du Rapport culturel ambitionnent de bouleverser les relations sociales par l'avènement d'une «culture nouvelle " incarnant les masses laborieuses. Dans une optique universaliste, cette «culture nouvelle» se doit d'être « nationale » et « populaire ». Pour l'AGEG, faire acte de scission face au bloc historique bourgeois implique un appel à l'union du peuple guadeloupéen, devant reposer sur l'alliance fondamentale des classes les plus exploitées et les plus fortes numériquement : la classe ouvrière et la paysannerie pauvre. Au sein de cette alliance de classes, c'est aux ouvriers d'exercer l'hégémonie : «la bourgeoisie s'efforce de maintenir son système culturel tandis que le prolétariat lutte pour le renverser (en même temps que la base économique) et pour édifier dans la société nouvelle une culture à caractère socialiste ${ }^{19}{ }^{\prime}$.

\section{Le gwoka au fondement d'un imaginaire national guadeloupéen}

Dans les années 1970, les ouvriers agricoles et les paysans pauvres se retrouvent autour d'un ensemble de pratiques musicales et dansées parmi lesquelles le quadrille, la biguine et le gwoka. L'ethnomusicologue Marie-Céline Lafontaine indique que ces pratiques étaient intimement liées, les mêmes musiciens passant facilement de l'une à l'autre ${ }^{20}$. Les rédacteurs du Rapport culturel analysent ce terrain musical comme le reflet des rapports sociaux construits à partir de stratégies hégémoniques spécifiques à l'ancienne société coloniale.

Ainsi, les rédacteurs du Rapport culturel de l'AGEG rejettent le quadrille parce que, selon eux, c'est la danse des asservis qui ont accepté leur condition servile et renoncé à la lutte ${ }^{21}$. La biguine, quant à elle, est reconnue comme une création guadeloupéenne. Toutefois les expositions coloniales ont construit auprès des Européens une image dégradante d'une Guadeloupe "de sucre et de vanille ${ }^{22}$ », très éloignée de la réalité antillaise. De plus, les mélodies de biguines se réfèrent au système tonal, ce qui va à l'encontre de l'idéologie nationaliste pour laquelle la tonalité est un symbole de la culture européenne.

Dans la continuité de cette analogie entre musique et idéologie, le gwoka ${ }^{23}$ est décrit comme une pratique répondant en tout point au projet politique indépendantiste, nationaliste, anticolonial et anticapitaliste. Selon les rédacteurs du Rapport Culturel en effet, le gwoka est une adaptation locale de pratiques musicales africaines qui ont su résister aux forces impérialistes françaises pour devenir la seule véritable musique populaire guadeloupéenne ${ }^{24}$, et qui a été «conservé dans toute sa pureté au sein des masses laborieuse ${ }^{25} »$. En outre, les contours mélodiques des chants gwoka ne renvoyant pas à l'échelle tonale, les rédacteurs du Rapport culturel les ont déclarés musique atonale, " c'est-à-dire qu'[ils] n'ont aucun centre de gravité tonal ${ }^{26}$ ».

Cette réflexion sur le rôle de la musique dans la construction d'une nation a par ailleurs abouti à la théorisation d'un concept musical appelé «gro-ka moderne ». Ce concept musical a été forgé par le guitariste de jazz Gérard Lockel ${ }^{27}$, qui écrit dans le livret de 
son premier album paru en 1976: "cette musique n'a rien à voir avec les lois fondamentales de la science musicale classique occidentale [...]. C'est une musique d'improvisation, qui se développe à partir d'un mode, mode qui donne naissance à une forme de mélodie, qui exprime le sentiment musical du peuple guadeloupéen ${ }^{28}$ ».

Si l'utilisation du concept d'atonalité par les rédacteurs du Rapport culturel ne renvoie pas à la deuxième école de Vienne et au développement de la musique sérielle, elle permet aux militants indépendantistes de marquer une différence et d'exprimer une radicalité musicale. Dans Philosophie de la nouvelle musique, Theodor Adorno écrit que « la musique radicale n'a pas réagi autrement contre la dépravation commerciale de l'idiome traditionnel; elle a été l'antithèse de l'industrie culturelle qui envahissait son domaine ${ }^{29}$ ». En Guadeloupe, le concept musical de "gro-ka moderne » a été pensé comme étant l'antithèse, non pas de l'industrie culturelle, mais de l'assimilation culturelle. Cette politisation du matériau sonore a orienté les interprétations relatives aux conduites musicales. Dans ce contexte, la pratique d'une musique est perçue comme un vecteur de domination (le quadrille), comme une caractéristique de l'assimilation (la biguine) ou comme un acte d'émancipation (le gwoka).

Support d'une pensée politique qui ne doit pas se limiter aux luttes économiques et sociales, le gwoka est ainsi devenu le symbole culturel du projet indépendantiste du camp patriotique regroupant un ensemble d'individus, de syndicats, d'associations et de groupes politiques engagés pour promouvoir une Guadeloupe indépendante et souveraine.

La couverture de ce Rapport culturel illustre bien l'avenir souhaité pour le corps économique et social.

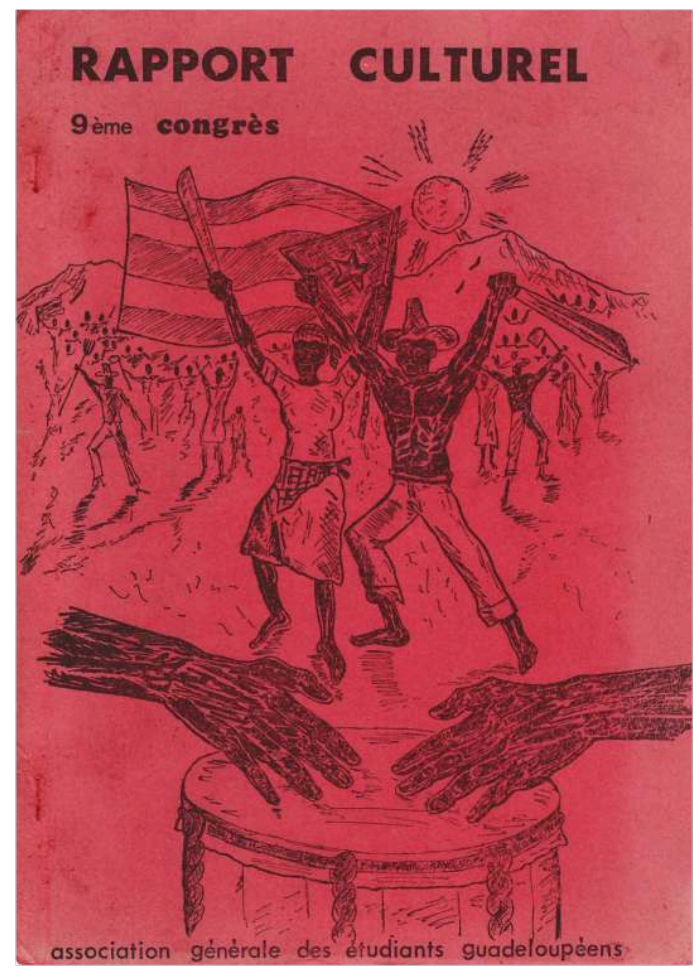

III. 1 : Couverture du Rapport culturel, AGEG, 1970 (collection privée)

Elle montre au premier plan un tambour ka. Là où, normalement, on aurait dû voir le torse du joueur de tambour ka (dont on ne voit que les mains), on voit une foule 
nombreuse de personnes arborant des outils caractéristiques du monde rural/agricole (machettes, houes, fourches...). Cette foule semble descendre de la montagne située en arrière-plan, montagne qui dans l'imaginaire caribéen renvoie aux esclaves fugitifs (les Marrons) qui se cachaient sur les hauteurs boisées difficiles d'accès. À l'avant de cette foule se tiennent côte à côte un homme et une femme portant la tenue de travail des ouvriers agricoles (pantalon aux jambes retroussées et chapeau bakoua pour l'homme, une gol avec un madras noué à la ceinture pour la femme). Ils tiennent tous les deux une machette et la femme arbore un drapeau symbole de la nation guadeloupéenne en devenir. Tous ont les bras levés. Est-ce un signe de victoire ou de préparation au combat? Ce qui est certain, c'est qu'il s'agit d'ouvriers agricoles guadeloupéens en lutte et déterminés à prendre le pouvoir. La couverture apparaît sur fond rouge, couleur des paysans bolchéviques, de la révolution culturelle ou encore de la révolution cubaine. Posé au premier plan de cette couverture, le tambour ka est pensé comme catalyseur de la révolution. Un soleil levant annonce des lendemains qui chantent.

Les rassemblements collectifs autour du gwoka sont ainsi devenus des lieux où s'est exprimée une volonté de transformer les rapports de pouvoir et de domination. Ces rassemblements rendent compte d'une localisation de la lutte politique imbriquée dans le champ culturel explicitement formulée par les étudiants de l'AGEG : « nous exigeons l'unité dialectique du politique et de l'artistique ${ }^{30} »$. Le besoin impératif de se différencier "politiquement » de l'ancienne puissance coloniale ${ }^{31}$ ne s'exprime pas de la même manière dans les îles caribéennes anglophones. Indépendantes, ces îles ont développé des cultures politiques créoles qui se caractérisent par un «investissement des institutions par des attitudes et des représentations formées dans l'expérience du passé esclavagiste et colonial ${ }^{32}$ ", ayant abouti à "l'émergence des nationalismes antillais [qui se] dotent de deux figures indissociables : celle de la contestation de l'ordre existant et celle de la fascination pour cet ordre et ses formes ${ }^{33}$ ".

En Guadeloupe, l'expérience insatisfaisante de la départementalisation (avec notamment le non-respect des idéaux de justice sociale qui avaient été annoncés) a nourri l'émergence d'un nationalisme plus radical exprimant un point de vue critique sur le pouvoir, un pouvoir qui ne suscite plus aucune attente. Pour se distancier des institutions politiques françaises, les pratiques culturelles associées au gwoka ont été investies et transformées en une agora permettant aux acteurs de déployer leur capacité d'agir en dehors de la sphère politique institutionnelle. Le gwoka a ainsi exercé un rôle nodal dans la fabrication d'un imaginaire national.

\section{Premiers efforts de mise en patrimoine du gwoka}

Parmi les objectifs que s'était fixée l'AGEG pour contribuer à la lutte culturelle du peuple guadeloupéen, figurait « la préservation du patrimoine culturel guadeloupéen et en particulier des traditions orales ${ }^{34} »$. Dans cette perspective, des actions de mise en valeur du gwoka se multiplient dans le but de lutter contre l'assimilation culturelle en faisant connaitre et en préservant ce " patrimoine culturel guadeloupéen ». Ainsi, au cours des années 1970, les rassemblements et les manifestations du camp patriotique s'organisent sur fond de musique ka et incluent presque toujours un léwoz ${ }^{35}$ dit «patriotique ». Afin de pallier le bannissement du gwoka des scènes officielles, des membres du camp patriotique lui offrent un espace d'expression en créant le Festival Gwoka de Sainte-Anne (FGK) dans les années 1980. Les membres du camp patriotique 
créent aussi des écoles, élaborent des méthodes d'apprentissage du tambour ka et produisent des essais d'historiographie. Grâce à ce type d'actions, le camp patriotique parviendra à changer le regard que les Guadeloupéens portent sur le gwoka.

C'est suite à ces actions que le gwoka, pratique marginale, socialement stigmatisée, associée à la ruralité agricole, a été valorisé, dans le but de construire une identité culturelle complémentaire d'une identité politique. Cette valorisation du gwoka a participé de la construction d'identités nouvelles, politisées, autour d'un projet indépendantiste. Renvoyant aux tribulations passées, tout en inscrivant le collectif dans un devenir commun, le gwoka a aussi été le support de la construction d'une identité/altérité par l'affirmation d'une ascendance africaine. Ainsi, pour les militants indépendantistes, le gwoka doit servir à représenter la classe subordonnée sur les plans économique, identitaire, culturel et politique en produisant des actions, des pratiques, des idées, rejoignant sur ce point les concepts développés par Michel Foucault quant à la dimension positive du pouvoir "producteur de subjectivités dans tout type de rapport social ${ }^{36} »$.

Dans les années 1980, avec l'élection de François Mitterrand à la présidence de la République française et l'arrivée de Jack Lang au Ministère de la Culture, les activités $\mathrm{du}$ camp patriotique entrent en déclin, après une brève période d'embrasement toutefois ${ }^{37}$. Dès son arrivée au pouvoir François Mitterrand élabore une action énergique pour contenir les volontés des séparatistes régionaux. Sur le plan culturel, Jack Lang met en place la nouvelle politique du Ministère de la Culture qui permet notamment «à tous les Français de cultiver leur capacité d'inventer et de créer, d'exprimer librement leurs talents et de recevoir la formation artistique de leur choix ; de préserver le patrimoine culturel national, régional ou des divers groupes sociaux pour le profit commun de la collectivité tout entière ${ }^{38} »$. François Mitterrand annonce que la nation est faite de régions parmi lesquelles les Outres-mers, que chacune a sa spécificité, encourageant ainsi l'usage des expressions culturelles régionales.

Ces nouvelles orientations sonnent le glas de la lutte politique ouverte des séparatistes qui se reportent, de manière plus forte encore, sur le «front culturel». Les acteurs guadeloupéens se réapproprient les nouvelles directives de la politique culturelle nationale pour continuer leur action subversive consistant à " décoloniser les esprits " et ce, sans risque d'être inquiétés par les autorités étatiques. L'action politique se réinvente à l'aune de ce nouveau contexte, en se dissimulant dans l'action culturelle.

En Guadeloupe, les nouvelles directives culturelles s'appliquent lentement. Les responsables d'espaces culturels continuent de refuser l'accès à la scène aux musiques non européennes, et notamment au gwoka. C'est dans ce contexte d'hostilité culturelle héritée de la période coloniale ${ }^{39}$ que le tournoi annuel organisé sur la plage par le Comité d'Animation Sportive et Culturelle (CASC), une association formée à SainteAnne en Guadeloupe à la fin des années 1980, se transforme en FGK en 1988. Ce festival prend de l'ampleur et devient le rendez-vous de tous les musiciens de gwoka de la Guadeloupe et un espace où les anciens militants indépendantistes aiment à se retrouver pour poursuivre leur travail, qu'ils qualifient de décolonisation des esprits. Organisé chaque année durant trois semaines au mois de juillet, ce festival offre alors aux Guadeloupéens un espace d'expression qui échappe au contrôle de la région et de l'État et qui permet de mettre en pratique les idées du mouvement indépendantiste relatives au gwoka et à la culture guadeloupéenne avec, notamment, des débats qui se 
tiennent exclusivement en langue créole. Le FGK contribue ainsi à faire connaître, à transmettre et à préserver le " patrimoine culturel guadeloupéen ».

\section{Introduction de la notion de « Patrimoine Culturel Immatériel » en Guadeloupe}

Illustrant la notion de retournement du stigmate conceptualisée par le sociologue américain Erwin Goffman, les militants du camp patriotique ont provoqué la réinvention des représentations associées à la pratique gwoka. Autrefois qualifié de mizik a vyé nèg (musique des gens déconsidérés), le gwoka est devenu dans les années 1990 une pratique partagée, reconnue et valorisée par une majorité de Guadeloupéens. Parallèlement, l'insertion du gwoka dans l'industrie musicale (maisons de production, concerts, festivals, presse spécialisée...) et le développement d'une production discographique support d'une création musicale fourmillante ont apporté une reconnaissance nationale et internationale au gwoka, qui a acquis dans les représentations individuelles et collectives un statut de «patrimoine culturel guadeloupéen ${ }^{40} »$.

Aussi, lorsque la notion de PCI est introduite pour la première fois en 2004 en Guadeloupe, elle arrive dans un contexte où la réflexion autour de la patrimonialisation est déjà bien avancée et où des actions de mise en patrimoine sont déjà en cours. C'est dans ce contexte que Félix Cotellon, Président du Festival Gwoka de Sainte-Anne, invite Chérif Khaznadhar, l'un des pères fondateurs de la Convention de l'UNESCO pour la sauvegarde du PCI, à présenter cette notion au public du festival. M. Khaznadar s'exprime deux fois en l'espace de quelques jours sur ce sujet. La première prise de parole, formelle, a lieu devant un public varié de porteurs de tradition, d'anciens acteurs du camp patriotique et aussi de simples badauds. La seconde, plus courte et informelle, est donnée au cours d'un rassemblement festif sur les terres de la famille Geoffroy, famille représentative de la culture et de la tradition locales.

Ainsi, avant même que la France ne ratifie la convention en 2006, la notion de PCI a été introduite en Guadeloupe, suscitant l'intérêt des organisateurs du FGK. Se saisissant de cette notion de PCI avant que l'État français n'adopte les dispositifs instaurés par la Convention UNESCO, il s'agit pour eux d'utiliser les outils mis à disposition par le texte international pour construire ce "nouveau régime de patrimonialité ${ }^{41}$ " à partir de catégories locales et vernaculaires.

Le FGK servira de première « arène de traduction ${ }^{42}$ » de la notion de PCI en Guadeloupe. Ce festival était à l'origine un simple tournoi de football, au sein duquel la musique s'est rapidement imposée. Il y a d'abord eu un bal de clôture, puis des concerts de gwoka modèn $^{43}$ et quelques koutanbou (rencontres de gwoka) organisés en contrepoint des matchs. En moins de dix ans, le sport s'est complètement effacé au profit de conversations sur l'identité et la culture guadeloupéennes et de spectacles de musique et de danse gwoka sous toutes ses formes, comme nous l'avons mentionné précédemment. Ces conversations, kozé en créole guadeloupéen, sont devenues un moment incontournable en même temps qu'une caractéristique majeure du festival. Organisés les après-midis, ces kozé sont menés par des musiciens et danseurs gwoka, des universitaires, des représentants d'organismes culturels et, plus rarement, des élus à la culture. Ils sont suivis d'un échange, ou bokantaj, avec le public. Relié à l'actualité 
culturelle, le thème choisi pour chaque kozé-bokantaj (causerie-débat) reflète cette même volonté de décolonisation des esprits évoquée plus haut. Entre 2004 (première présentation de la Convention UNESCO en Guadeloupe) et 2011 (lancement du projet d'inscription du gwoka sur la LR du PCI), chaque édition du festival a proposé au moins un kozé-bokantaj sur la place et la fonction du gwoka dans la société guadeloupéenne.

Parallèlement à l'organisation de ces kozé-bokantaj, un Centre des Musiques et Danses Traditionnelles et Populaires (CMDTP) est créé en 2005. Présidé par Félix Cotellon, ce centre reprend et amplifie un projet que le Comité d'Animation Sportive et Culturelle (CASC) avait lancé à la fin des années 1990. Ce projet, intitulé Les Voix du Patrimoine, visait à établir une vision plus juste de la culture guadeloupéenne à travers un inventaire de tous les éléments constitutifs de la musique et de la danse que les Guadeloupéens estimaient leur appartenir. Au moment de la création du CMDTP, le CASC avait déjà fait procéder à deux collectes. L'une était relative aux chants et aux danses du répertoire indo-guadeloupéen. L'autre était consacrée aux chants de marins de La Désirade. Deux autres collectes sont effectuées par l'ethnomusicologue Dominique Cyrille, en 2006 sur les quadrilles et en 2007 sur les bèlès et les chants de travail de la terre.

$\mathrm{Au}$ mois de septembre 2008, après l'embauche d'un directeur et de Dominique Cyrille, responsable de la mission Patrimoine, le CMDTP entre réellement en activité. Il est alors rebaptisé Centre Rèpriz et commence une nouvelle phase d'activités en vue de la sauvegarde et de la promotion de la musique et de la danse de Guadeloupe.

\section{Traduction locale et mise en œuvre de la notion de Patrimoine Culturel Immatériel en Guadeloupe}

33 C'est dans ce contexte qu'un certain nombre d'actions ont été conduites en " connectant des échelles différentes de gouvernance ${ }^{44}$ » qui se sont rencontrées de manière formelle dans le cadre de séminaires. En 2003 et 2005, le CASC s'associe à la médiathèque Bettino Lara de Guadeloupe (LAMECA) pour proposer un Séminaire d'Ethnomusicologie Caribéenne. Ce séminaire réunit des chercheurs internationaux spécialistes de la musique, de la danse et de l'histoire des pays du circum-Caraïbe pour discuter de problématiques communes à toutes les musiques au tambour de la Caraïbe et rappeler l'ancrage de la Guadeloupe dans son espace géoculturel naturel. En 2007, le Centre Rèpriz se joint à l'organisation de ce séminaire qui a pour thématique: Sauvegarder et promouvoir les musiques caribéennes; Enjeux et problématiques nouvelles. Cette édition est consacrée à la convention UNESCO présentée dans le cadre du séminaire par Christian Hottin (chef de la mission ethnologie du Ministère de la Culture et de la Communication). Illustrant les propos de Chiara Bortolotto sur la localisation du global qui «devient saisissable dans les branchements où les différents acteurs humains (diplomates, fonctionnaires, experts, etc.) et non humains (Convention, formulaires, etc.) se rencontrent et produisent les traductions nécessaires à leurs interactions ${ }^{45}$ », l'édition 2007 du Séminaire d'Ethnomusicologie Caribéenne fait se rencontrer des responsables d'associations culturelles guadeloupéens, martiniquais et bretons, un représentant du Ministère de la Culture (Jean-Pierre Estival, inspecteur DMDTS) qui intervient sur la mise en œuvre de la Convention, un représentant de la Direction Régionale des Affaires Culturelles (DRAC ${ }^{46}$ ) Guadeloupe (Philippe Bon), le président de la commission culturelle du Conseil général de la Martinique (Marcellin 
Nadeau), le président de la commission culture du Conseil régional de Martinique (Edmond Mondésir), des représentants des commissions culture des conseils régional et général de Guadeloupe, le directeur du Bureau de l'UNESCO à la Jamaïque, un représentant du Ministère de la Culture de la République dominicaine, le représentant de la culture à la Dominique, le représentant du Folk Research Center de Sainte-Lucie, des universitaires (venus des États-Unis, de Cuba, de Colombie, du Vénézuela). Suite à ce séminaire, le Centre Rèpriz devient la première ONG française accréditée par l'UNESCO pour la Sauvegarde du PCI.

Ainsi, avant l'intégration complexe de la norme internationale aux dispositifs institutionnels et législatifs français ${ }^{47}$, la notion de PCI a été investie localement en Guadeloupe, produisant des espaces où elle a été discutée, encodée et décodée. Par ailleurs, l'appropriation du concept de PCI par les acteurs culturels guadeloupéens s'est traduite par une série d'actions qui ont permis de construire un cadre adapté à la mise en œuvre de la Convention UNESCO et de mettre en place « des structures chargées de ce domaine ${ }^{48} »$. À la différence de ce qui a pu être observé ailleurs ${ }^{49}$, ces structures n'ont pas été mises en place par les institutions de l'État dédiées à la Convention et il n'y eut ni recrutement de fonctionnaires ni formation d'experts. En Guadeloupe, la mise en œuvre des instruments normatifs internationaux et leur inscription dans les catégories patrimoniales de la France se sont appuyées sur des pratiques locales préexistantes (festival, kozé-bokantaj, séminaires...). Ces pratiques locales ont influencé les définitions des catégories "globales » et déterminé la déclinaison concrète des instruments normatifs internationaux au regard des spécificités du terrain, démarche qualifiée d'exemplaire par le Secrétariat de la Convention au moment de l'inscription. Les circulations ont donc été établies de l'UNESCO vers les pratiques culturelles guadeloupéennes et ce sont les acteurs culturels locaux qui ont été à l'initiative de la mise en relation du global, du national et du local.

Ainsi, le projet d'inscription du gwoka sur la liste représentative du PCI de l'UNESCO a été impulsé par Félix Cotellon. Avocat de profession, bâtonnier de 2005 à 2007, Félix Cotellon est aussi un militant indépendantiste de la première heure, "un ancien de l'AGEG » qu'il a présidée au milieu des années 1970. Il lance le projet d'inscription au mois de juillet 2011 dans le cadre du Festival Gwoka de Sainte-Anne. Comme on l'a vu, ce projet s'inscrit dans la continuité de plusieurs kozé-bokantaj organisés entre juillet 2004 et mai 2011 pour sensibiliser et faire connaître la Convention pour la sauvegarde du PCI et ses enjeux aux Guadeloupéens. Dans sa mise en œuvre, le projet d'inscription est porté par l'ONG Rèpriz dont Félix Cotellon est le président fondateur.

Pour répondre aux exigences de la Convention UNESCO et garantir la participation effective de la communauté gwoka et son "consentement éclairés ", un comité «Lyannaj pou Gwoka » est formé en 2011. Rassemblant une trentaine de porteurs de la tradition gwoka, ce comité travaille à l'élaboration du dossier de candidature entre 2011 et 2014, en se scindant en cinq cellules ayant chacune des missions précises: identification, sauvegarde, communication/information, documentation, budget.

Après l'annonce officielle du projet d'inscription au mois de juillet 2011, des associations de gwoka de Guadeloupe et d'Ile-de-France, des musiciens, danseurs et chanteurs, des chercheurs universitaires, des responsables de lieux de diffusion et des membres de la société civile s'impliquent aux côtés du comité «Lyannaj pou Gwoka ». La cellule "information» du comité intervient dans des léwoz à la demande d'associations et organise une trentaine de réunions avec les membres de la société 
civile, les conseils municipaux, les instances départementales et régionales. De septembre 2011 à février 2012, les membres des cellules "identification", «documentation» et «sauvegarde» tiennent des réunions hebdomadaires pour préparer le dossier de candidature. Pendant cette période, le «Lyannaj pou Gwoka » se retrouve chaque mois en réunion plénière pour coordonner les différentes actions entreprises. Par ailleurs, deux léwoz sont organisés par le «Lyannaj pou Gwoka » pour expliquer l'intérêt du projet d'inscription et faire circuler une pétition de soutien à ce projet. Intitulée "An Dakò, je signe la pétition", elle fait également l'objet d'une diffusion en ligne. Au mois de mars 2012, elle a déjà recueilli 2081 signatures (parmi lesquelles figurent 347 de chanteurs, danseurs et joueurs de tambour ka), des attestations de consentement d'associations, des délibérations de collectivités locales, au moment même où le candidat à la présidentielle François Hollande prononce un discours aux Outres-mers dans lequel il indique soutenir « l'initiative tendant à classer le gwoka au patrimoine de l'humanité51 ».

\section{Conflits autour du projet d'inscription}

Le processus d'inscription a provoqué une mise sous tension de la société guadeloupéenne, en activant simultanément des rhétoriques identitaires et politiques. Cette mise sous tension du champ social a notamment pris la forme d'un conflit opposant deux groupes: le "Lyannaj pou Gwoka " et le "Kolektif pou Gwoka ", constitué en octobre 2011 pour contester le projet d'inscription. Ces deux groupes, représentés par des acteurs ayant en commun un passé de militants indépendantistes, ont débattu avec véhémence de la nécessité d'inscrire le gwoka sur la LR de l'UNESCO. Ces prises de position ont été énoncées dans différents contextes: situations quotidiennes, réunions publiques, débats sur les radios et chaînes de télévision, presse écrite. Ainsi, le quotidien régional France-Antilles titrait son édition du 14 novembre 2011, en créole: "Chiraj alantou gwoka». Un sous-titre en français précisait: «la demande d'inscription du gwoka au patrimoine culturel immatériel de l'humanité de l'UNESCO crée la polémique ». La deuxième page du quotidien consacrait un article à cette "polémique autour du patrimoine » en rapportant les propos des représentants de chacun des deux "camps ». Pour Félix Cotellon, "en inscrivant le gwoka au patrimoine de l'UNESCO, c'est une manière d'enraciner cette musique en Guadeloupe ${ }^{52}$ ». Pour Jean-Claude Nelson, opposé au projet d'inscription, «le gwoka sera soumis à des règles dictées par la France ${ }^{53}$ ».

Cette divergence quant aux usages politiques de l'identité culturelle a structuré l'opposition entre les deux camps. Pour les Guadeloupéens qui y étaient favorables, l'inscription du gwoka avait pour finalité d'agir sur les relations de pouvoir en garantissant la mise en œuvre par les collectivités et/ou l'État français de politiques publiques de soutien au gwoka. En s'appropriant les compétences du pouvoir culturel, elle était aussi l'occasion de contourner l'État pour se dire en tant que Guadeloupe sur la scène internationale, comme le chante Wozan Monza dans un morceau intitulé « Gwoka UNESCO » : « je suis comme les peuples Kurde et Palestinien, une minorité qui est sans rien, pas besoin d'un État pour exister, ils m'ont reconnu, dans le concert de l'humanité, je suis un patrimoine immatériel, un petit morceau de l'humanité, je ne vends pas mon âme au diable pour rien, je suis la musique gwoka guadeloupéenne ${ }^{54}$ ». Pour les Guadeloupéens opposés à l'inscription, le statut institutionnel actuel de la 
Guadeloupe était inconciliable avec ce projet d'inscription "sur la liste du colonisateur » et leur faisait craindre un risque de dépossession culturelle.

Dans un contexte où les Guadeloupéens ont manifesté leur volonté de rester dans le cadre de la République française, à l'occasion notamment du référendum du 7 décembre 2003, et où la grande majorité des organisations anticolonialistes ont aujourd'hui un projet politique institutionnel évoluant dans le cadre de la République française, les tensions identitaires suscitées par une inscription du gwoka guadeloupéen sur la liste du patrimoine français ont montré le déploiement de conduites politiques imbriquées dans le champ culturel.

41 Le projet d'inscription du gwoka sur la LR du PCI, porté par un ancien militant de l'AGEG, a été perçu par une partie des acteurs comme la suite logique de l'action que le camp patriotique avait formulée dans le Rapport culturel. Dans cette perspective, l'inscription du gwoka sur la LR du PCI devenait une manière d'accéder à « la sphère de l'hégémonie et des rapports éthico-politiques ${ }^{55}$ ». Ainsi, le gwoka a d'abord été valorisé en tant que pratique sociale et culturelle attachée à une branche économique donnée (ouvriers et paysans), puis en tant que pratique attachée à une culture de classe, ensuite en tant que projet politique indépendantiste/nationaliste, et enfin en tant que " pratique culturelle [représentative] de l'identité guadeloupéenne ${ }^{56}$ ». Ce processus acte la refonte du paysage idéologique et culturel guadeloupéen, réalisant la «mutation morale et cognitive de la conscience sociale ${ }^{57}$ » souhaitée par le Rapport culturel. Sur le plan politique, cette reconnaissance hégémonique indique qu'un renversement s'est opéré et que le groupe social qui porte cette pratique a désormais une fonction dirigeante ${ }^{58}$ dans l'exercice du pouvoir en participant (même indirectement) à la mise en œuvre des politiques culturelles de soutien et de valorisation du gwoka au sein des institutions de l'État (Ministère de la culture, Conseil régional, Conseil général).

Du côté des opposants au projet d'inscription, cette démarche a été perçue comme une trahison de l'action que le camp patriotique avait formulée dans le Rapport culturel. Pour ceux-là, cette inscription a fait naître la crainte de voir ce long combat politique et culturel se dissoudre dans l'universel. En faisant du "patrimoine culturel guadeloupéen », catégorie définie par le camp patriotique, un patrimoine culturel immatériel de l'humanité inscrit sur la liste de l'ancienne puissance colonisatrice, l'inscription participait au renforcement du pouvoir hégémonique de la France. Réactivant les traumatismes de l'assimilation culturelle liés aux effets pervers de la départementalisation, cette inscription a fait revenir le spectre d'une possible négation de la spécificité de l'identité culturelle guadeloupéenne. Cette substitution du terme " guadeloupéen » par « immatériel» dans la qualification du patrimoine culturel a pu être vécue comme une remise en cause du combat politique mené depuis les années 1960 pour la construction d'un État-nation guadeloupéen.

Le débat a ainsi verbalisé des positionnements stratégiques politiques différents autour de l'identité culturelle partagée. Ce processus dynamique d'appropriation internationale et de réappropriation communautaire a aussi montré que le gwoka continue d'être associé, pour l'ensemble des acteurs, à un discours de contestation face à la France. Les acteurs étaient en désaccord sur la manière de se dire et de s'opposer à l'État français.

44 Par-delà l'adhésion à l'une ou l'autre des propositions, ce sont les trajectoires individuelles qui ont déterminé le vécu que les individus ont eu du processus d'inscription et/ou leurs prises de position : pour les uns c'était l'aboutissement de la 
lutte culturelle (débutée avec le mouvement indépendantiste), pour les autres c'était une forme de reniement. C'est peut-être pourquoi les prises de position se sont avérées fluctuantes, parfois difficiles à saisir, confirmant l'analyse du politologue martiniquais Justin Daniel qui souligne « la capacité des acteurs sociaux à recourir simultanément ou alternativement à plusieurs registres ou répertoires d'action, tout en accréditant l'idée qu'ils partagent un système de valeurs commun ${ }^{59}$ ». Cette ambiguïté est le reflet d'une attitude critique vis-à-vis de l'appartenance à l'ensemble français, attitude qui s'est développée de manière spécifique dans cette société post-esclavagiste/postcoloniale où le processus de décolonisation a été sapé par la départementalisation puis par l'arrivée de la France des régions. La capacité d'agir et d'être Guadeloupéen a été diluée dans le vaste ensemble français, marginalisant le potentiel politique subversif des acteurs. Aussi, pour certains, le processus d'inscription a réactivé cette crainte d'être à nouveau, au mieux trompés, au pire dépossédés, d'une pratique culturelle fondatrice de leur identité.

\section{Le gwoka : une arène patrimoniale}

Le débat intense entre le «Lyannaj pou Gwoka » et le "Kolektif pou Gwoka » a duré environ huit mois. Au mois de novembre 2011, le décès du chanteur Hilaire Geoffroy, figure du gwoka, respectée et connue de tous, a contribué à tempérer les esprits. Symbole fort s'il en est, des représentants importants des deux groupes opposés ont chanté ensemble l'adieu traditionnel "Dòmi byen» (Dors bien) au moment de la fermeture du cercueil. Ainsi, lorsque le dossier d'inscription préparé par le «Lyannaj pou Gwoka " a été présenté dans sa version définitive au Ministère de la Culture en mars 2013, les dissensions ne s'exprimaient plus de manière vive, même si chaque groupe restait sur ses positions. C'est en cela que le gwoka est devenu un espace politique d'affrontement à l'intérieur de la communauté des gens du gwoka. Il est devenu une « arène patrimoniale ${ }^{60}$ ». Cette notion est empruntée à Olivier Givre qui la décrit ainsi : «la notion d'arène patrimoniale permet d'appréhender les processus patrimoniaux comme des espaces de savoir/pouvoir (Foucault), dans lesquels se déploient des stratégies et des tactiques (de Certeau) portant sur la légitimité à définir et user d'objets patrimoniaux ${ }^{61}$ ».

En effet, en Guadeloupe, cette arène patrimoniale a été un espace où se sont déployées des stratégies quant à l'usage politique du gwoka en tant que bien culturel. Dans un article paru en 2012, Berardino Palumbo écrit que «si chacun des États peut avoir plusieurs biens patrimoniaux qui le représentent, chaque bien doit, quoi qu'il en soit, pouvoir être attribué sans équivoque à un État unique ${ }^{62}$ ». Pour ce qui est de la France, les biens patrimoniaux qui la représentaient alors étaient, entre autres, «le repas gastronomique français » (2010), "le savoir-faire de la dentelle au point d'Alençon » (2010), "l'équitation de tradition française» (2011), "les ostensions septennales limousines " (2013). Si, vus de la Guadeloupe, ces biens patrimoniaux pouvaient être attribués à la France "sans équivoque ", pour une majorité de Guadeloupéens il ne pouvait en être de même pour le gwoka. Pour eux, en effet, le gwoka ne peut en aucun cas exprimer des « qualités nationales » françaises ${ }^{63}$. Il renvoie à une territorialité et à une historicité distinctives et spécifiquement guadeloupéennes. C'est pourquoi envisager de faire du gwoka un bien culturel français par le biais de l'inscription a suscité les vives discussions mentionnées plus haut. Si les deux groupes qui se sont 
opposés dans le cadre du processus d'inscription s'accordaient sur le fait que le gwoka ne pouvait pas être une "icône de l'identité nationale " française ${ }^{64}$, pour ceux qui soutenaient le projet d'inscription, il s'agissait de faire exister la Guadeloupe sur la scène internationale en tant que territoire. Cette démarche était pour eux l'équivalent de l'inscription de l'église de la Nativité à Bethléem, qui a permis de faire reconnaitre à l'international l'existence de la Palestine en tant qu'entité politique et culturelle, indépendamment de son rattachement à l'État d'Israël. Pour ceux qui étaient opposés à l'inscription, faire du gwoka un bien culturel français était contraire à leur conception du politique. Pour eux, l'inscription ne permettait pas de produire des subjectivités émancipatrices mais maintenait et renforçait sur la scène internationale leur statut de territoire non indépendant de la France.

Le gwoka n'a pas fait exception puisque, comme l'ont montré d'autres travaux ${ }^{65}$, il y a toujours des tensions et/ou conflits, dès que l'intention d'inscription sur une liste patrimoniale est manifestée, que le patrimoine soit matériel ou immatériel. Toutefois, dans le cas du baroque de la région de Noto $^{66}$, le conflit a porté sur la question de la désignation des villes et monuments concernés par le projet d'inscription. Dans le cas du samba ${ }^{67}$, les conflits sont nés entre les différentes écoles de samba quant au choix du type de samba à inscrire. S'agissant de la Guadeloupe, c'est l'usage politique du gwoka sur la scène internationale qui a cristallisé les tensions de cette arène patrimoniale.

L'exemple du gwoka montre aussi le potentiel de subjectivation et d'agency que permet la Convention. Les actions culturelles entreprises par le camp patriotique dès les années 1970 en Guadeloupe sont en effet entrées en résonance notamment avec les articles 11 à 15, qui portent sur la notion de sauvegarde du PCI. Les expériences acquises dans le cadre du mouvement patriotique guadeloupéen ont permis de tirer pleinement parti de "l'injonction participative ${ }^{68}$ » requise par la Convention. Le « consentement éclairé des communautés » a, dans cette perspective, eu une résonance politique et a contraint les individus à se positionner. Le processus de patrimonialisation tel qu'il s'est déroulé en Guadeloupe a ainsi rappelé que ni les groupes, ni les communautés ne sont homogènes, permanents et stables, contrairement à ce que l'article 15 de la Convention suggère.

En novembre 2014, le gwoka est inscrit sur la LR sous l'appellation «Le gwoka: musique, chants, danses et pratique culturelle représentatifs de l'identité guadeloupéenne ", une appellation délibérément choisie pour que la Guadeloupe soit immédiatement identifiée comme territoire d'origine de l'élément inscrit et qu'il n'y ait aucune ambiguïté quant aux détenteurs de ce patrimoine culturel, bien qu'il soit inscrit sous l'égide de la France.

La spécificité de ce processus d'inscription montre un exemple de communauté désireuse de s'affranchir du contrôle d'un État vécu comme une puissance hégémonique. Le cas de la Guadeloupe met en lumière un potentiel de la Convention peu étudié par la recherche, celui d'offrir aux minorités la possibilité de se définir sur la scène internationale en contournant le pouvoir de l'État auquel elles sont rattachées. 


\section{BIBLIOGRAPHIE}

ADORNO Theodor, Philosophie de la nouvelle musique, Paris, Gallimard, 1962 [1949], p. 15.

BORTOLOTTO Chiara (dir.), Le patrimoine culturel immatériel : enjeux d'une nouvelle catégorie, Paris, MSH, 2011.

BORTOLOTTO Chiara et BERLINER David, «Introduction : le monde selon l'Unesco », Gradhiva, $\mathrm{n}^{\circ} 18$, 2013, p. 4-21.

BORTOLOTTO Chiara, «L'Unesco comme arène de traduction : la fabrique globale du patrimoine immatériel », Gradhiva : revue d'anthropologie et d'histoire des arts, nº 18, 2013, p. 50-73.

CÉSAIRE Suzanne, « Misère d'une poésie », Tropiques, n 4, 1942, p. 48-50.

CHATTERJEe Partha, The Nation and its Fragments: Colonial and Post Colonial Histories, Princeton NJ, Princeton University Press, 1993.

CIAVOLELLA Riccardo et WITTERSHEIM Éric, Introduction à l'anthropologie du politique, Louvain-laNeuve, De Boeck, 2016.

CONSTANT Fred et DANIEL Justin, 1946-1996 : cinquante ans de départementalisation en outre-mer, Paris, L'Harmattan, 1997.

CYRILLE Dominique, «Popular Music and Martinican-Creole Identity », Black Music Research Journal, vol. 22, no. 1, 2002, p. 65-83.

CYRILLE Dominique, «The Politics of Quadrille Performance in 19th-Century Martinique ", Dance Research Journal, vol. 38, no. 1/2, 2006, p. 43-60.

DAhomay Marie-Line, "The Evolution of Gwoka from the Twentieth Century to the Present Day ", MELYON-REINETTE Stéphanie (ed.), Marronnage and Arts: Revolts in Bodies and Voices, Newcastle upon Tyne, Cambridge Scholars Publishing, 2012, p. 2-10.

DANIEL Justin, «L'espace politique aux Antilles françaises », Ethnologie française, vol. 32, $\mathrm{n}^{\circ} 4, \mathrm{p}$. 589-600.

DANIEL Justin, « Les formes d'expression politique dans la Caraïbe : ambivalence et paradoxe de la production de l'ordre politique », BERTHOMIÈRE William et CHIVALLON Christine (dir.), Les diasporas dans le monde contemporain : un état des lieux, Paris / Pessac, Karthala /MSHA, 2006, p. 361-376. GIVRE Olivier, « Savoirs et pouvoirs, stratégies et tactiques dans l'arène patrimoniale du Nestinartsvo (Bulgarie) », Civilisations, vol. 61, n 1, 2012, p. 103.

GRAMSCI Antonio, Cahiers de Prison, Paris, Gallimard, 1983 [1948-1951].

HolLANDE François, « Mes trente engagements pour les Outre-mers ", François Hollande 2012, p. 8 (georgespatient.fr/wp-content/uploads/2012/05/30-engagements-outre-mer.pdf, consulté le 7 juin 2019).

HOTTIN Christian, «L'ethnologie, un métier du patrimoine ? Réflexions autour de la question du patrimoine culturel immatériel », In Situ : revue des patrimoines, n 30, 2016.

LAFONTAINE Marie-Céline, Carnot par lui-même, Alors ma chère moi, Paris, Éditions Caribéennes, 1986. 
LOCKEL Gérard, « Gwoka Moden, musique de lutte de classes au service des ouvriers et des paysans guadeloupéens ", livret accompagnateur du disque Gérard Lockel ka présenté Gro Ka

Moden, Guadeloupe Disques, 1976.

MANUEl Peter (ed.), Creolizing Contradance in The Caribbean, Philadelphia, Temple University Press, 2009.

MARTIN Denis-Constant et CONSTANT Fred, Les démocraties antillaises en crise, Paris, Karthala, 1996, p. 13.

PALUMBO Berardino, «L'UNESCO et le campanile : patrimoine universel et patrimoines locaux », FABRE Daniel et IUSO Anna (dir.), Les monuments sont habités, Paris, MSH, 2010, p. 157.

SAMSON Guillaume, « Le maloya au patrimoine mondial de l'humanité : enjeux culturels, politiques et éthiques d'une labellisation », Cahiers d'ethnomusicologie, n² 24, 2011, p. 157-171.

SANDRONI Carlos, «L'ethnomusicologue en médiateur du processus patrimonial : le cas de la samba de roda », ВОRTOLOTTO Chiara (dir.), Le patrimoine culturel immatériel : enjeux d'une nouvelle catégorie. p. 233-252.

TURGEON Laurier, « Introduction : du matériel à l'immatériel. Nouveaux défis, nouveaux enjeux », Ethnologie française, vol. 40, $\mathrm{n}^{\circ}$ 3, 2010, p. 389-399.

\section{NOTES}

1. CONSTANT Fred et DANIEL Justin, 1946-1996: cinquante ans de départementalisation en outre-mer, Paris, L'Harmattan, 1997.

2. Nous traduisons " mizik a vyé neg " par "musique des gens déconsidérés " pour rester fidèle aux représentations négatives contenues dans l'expression vyé neg, héritée de la période coloniale. Vyé nèg est une expression du créole guadeloupéen. Elle est généralement employée pour désigner une personne le plus souvent native de la zone caribéenne, de la Martinique ou de la Guadeloupe notamment. Vyé nèg désigne un Antillais qui compte un ou plusieurs Africains réduits en esclavage parmi ses ancêtres. Jusqu'aux années 1980, le vyé nèg était perçu comme un individu ayant un mode de vie et des habitudes contraires à celles qu'on associait aux personnes dont l'éducation, le style de vie et les manières étaient jugées "propres » et agréables, voire civilisées. Le vyé neg était associé à la partie de la population la plus vile, des plantations et des faubourgs, celle qui vivait en marge de la «bonne société » guadeloupéenne.

Plus précisément, en créole guadeloupéen, vyé est encore aujourd'hui un adjectif à connotation péjorative qui s'applique à une chose indésirable, sans intérêt et, dans certaines situations, ridicule ou/et laide. À l'exception de vyé kò qu'on utilise pour parler d'une personne âgée, qu'on n'aime pas et qu'on trouve ridicule (pour la différencier d'une personne âgée qu'on appellera gran moun en signe d'amour et/ou de respect), vyé n'est pas lié à une notion d'ancienneté ou de vieillerie. Ainsi par exemple, vyé mannyè se traduit selon le contexte par " mauvaises mœurs » ou "manières désagréables "; vyé loto désigne une voiture sans aucune valeur, pas nécessairement vieille, mais qu'on estime moche et dans laquelle on ne voudrait pas être vu ; vyé rad fait référence à un vêtement laid, ridicule, mais pas forcément vieux ni ancien, etc.

Jusqu'aux années 1980, l'expression vyé nèg désignait une personne d'ascendance africaine à laquelle on ne voulait vraiment pas être associé. Dans les années 1970/80, les militants indépendantistes se sont attachés à déconstruire les représentations négatives véhiculées par l'expression vyé neg. Il en a résulté un retournement du stigmate qui permet aujourd'hui à la plupart des gens du gwoka de l'utiliser avec fierté pour revendiquer leur ascendance africaine. Plus largement, l'expression vyé neg est passée dans le langage courant. Elle peut être utilisée par 
tout guadeloupéen qui veut souligner la contribution des ancêtres africains à la société guadeloupéenne.

3. DANIEL Justin, «L'espace politique aux Antilles françaises », Ethnologie française, vol. $32, \mathrm{n}^{\circ} 4$, p. 589-600.

4. SAMSON Guillaume, "Le maloya au patrimoine mondial de l'humanité : enjeux culturels, politiques et éthiques d'une labellisation ", Cahiers d'ethnomusicologie, $\mathrm{n}^{\circ} 24,2011$, p. 157-171.

5. Ibid.

6. HotTin Christian, «L'ethnologie, un métier du patrimoine ? Réflexions autour de la question du patrimoine culturel immatériel », In Situ : revue des patrimoines, n 30, 2016.

7. Communication personnelle avec l'historien guadeloupéen Jean-Pierre Sainton, juillet 2018.

8. L'expression "porteurs de tradition » était utilisée en Guadeloupe avant que la convention UNESCO n'y soit présentée. Elle y a été introduite au début des années 2000 par des ethnomusicologues américains qui ont participé à des conférences publiques sur les musiques caribéennes en Guadeloupe entre 2002 et 2007. L'expression "porteurs de tradition » est en circulation depuis le tournant des années 2000 dans le monde académique américain sous l'appellation «tradition bearers ».

9. BORTOLOTTO Chiara, «L'Unesco comme arène de traduction : la fabrique globale du patrimoine immatériel », Gradhiva : revue d'anthropologie et d'histoire des arts, $\mathrm{n}^{\circ} 18,2013$, p. 50-73.

10. À l'origine, la départementalisation, ou assimilation administrative et économique dans l'ensemble français, avait été imaginée par les habitants des Outre-mer comme l'aboutissement des luttes pour l'égalité des droits entamée sous la Révolution. Dans les faits, le modèle assimilationniste d'intégration mis en place par l'État français s'est avéré être une forme de négation de l'histoire et de la culture des départements d'outre-mer. Sur cette question, voir notamment CONSTANT et DANIEL, 1946-1996 : cinquante ans de départementalisation en outre-mer.

11. AGEG, Rapport culturel, $9^{\mathrm{e}}$ Congrès, Paris, AGEG, 1970, p.65.

12. Ibid., p. 63.

13. Ibid., p. 14.

14. Ibid., p. 66.

15. Ibid., p. 54.

16. Ibid., p. 55 .

17. Ibid., p. 1.

18. Ibid., p. 14.

19. Ibid., p. 5.

20. LAFOnTAINE Marie-Céline, Carnot par lui-même, Alors ma chère moi, Paris, Éditions Caribéennes, 1986.

21. La recherche ethnomusicologique va à l'encontre de ces affirmations : MANUEL Peter (ed.), Creolizing Contradance in The Caribbean, Philadelphia, Temple University Press, 2009 ; CYRILLE Dominique, "The Politics of Quadrille Performance in 19th-Century Martinique", Dance Research Journal, vol. 38, no. 1/2, 2006, p. 43-60.

22. CÉSAIRE Suzanne, « Misère d'une poésie », Tropiques, ${ }^{\circ}$ 4, 1942, p. 48-50.

23. La réflexion sur la langue créole en était à ses balbutiements en 1970, aussi dans le Rapport culturel, le mot « gwoka » est orthographié « gro-ka ».

24. Daнomay Marie-Line, «The Evolution of Gwoka from the Twentieth Century to the Present Day », MELYON-REINETTE Stéphanie (ed.), Marronnage and Arts: Revolts in Bodies and Voices, Newcastle upon Tyne, Cambridge Scholars Publishing, 2012, p. 5.

25. AGEG, Rapport culturel, p. 14.

26. Ibid., p. 13.

27. Selon Jean-Pierre Sainton, le Rapport culturel de l'AGEG est directement issu des rencontres des étudiants avec Gérard Lockel, qui venait d'opérer son retour définitif en Guadeloupe et 
commençait ses enquêtes et sa conceptualisation du gwoka moderne. Communication personnelle avec l'historien guadeloupéen Jean-Pierre Sainton, juillet 2018.

28. LOCKel Gérard, «Gwoka Moden, musique de lutte de classes au service des ouvriers et des paysans guadeloupéens ", livret accompagnateur du disque Gérard Lockel ka présenté Gro Ka Moden, Guadeloupe Disques, 1976.

29. ADORNo Theodor, Philosophie de la nouvelle musique, Paris, Gallimard, 1962 [1949], p. 15.

30. AGEG, Rapport culturel, p. 62.

31. ChATTERJEe Partha, The Nation and its Fragments: Colonial and Post Colonial Histories, Princeton NJ, Princeton University Press, 1993.

32. MARTIN Denis-Constant et cONSTANT Fred, Les démocraties antillaises en crise, Paris, Karthala, 1996, p. 13.

33. Ibid., p. 19.

34. AGEG, Rapport Culturel, p. 67.

35. Le léwoz est un rassemblement festif nocturne au cours duquel on joue et on danse le gwoka.

36. CIAVOLELLA Riccardo et WITTERSHEIM Éric, Introduction à l'anthropologie du politique, Louvain-laNeuve, De Boeck, 2016, p. 151.

37. Dans les années 1983-1984, des attentats sont commis à Paris et en Guadeloupe par des membres du camp patriotique, aboutissant à l'emprisonnement par l'État français de certains responsables indépendantistes.

38. Article 1 du décret 82-394 du 10 mai 1982 relatif à l'organisation du Ministère de la Culture.

39. Voir à ce propos CYRILLE Dominique, « Popular Music and Martinican-Creole Identity ", Black Music Research Journal, vol. 22, no. 1, 2002, p. 65-83.

40. AGEG, Rapport culturel, p. 14.

41. TURGEON Laurier, "Introduction: du matériel à l'immatériel. Nouveaux défis, nouveaux enjeux », Ethnologie française, vol. 40, nº 3, 2010, p. 389-399.

42. BORTOLOTTO, «L'Unesco comme arène de traduction ».

43. Le gwoka modèn (orthographié «gro-ka moderne" dans le Rapport culturel) est un concept musical développé par Gérard Lockel qui a stimulé et nourri la créativité de nombreux musiciens guadeloupéens. Voir à ce propos CAMAL Jérôme, From Gwoka Modènn To Jazz Ka: Guadeloupean Music Between Nationalism And Creolization, Thèse de Musicologie, Washington University, St. Louis, États-Unis, 2011.

44. BORTOLOTTO, «L'Unesco comme arène de traduction », p. 70.

45. Ibid.

46. Antenne du Ministère de la Culture en région.

47. HOTTIN, «L'ethnologie, un métier du patrimoine?».

48. BORTOLOTTO, « L’Unesco comme arène de traduction », p. 65.

49. Ibid.

50. Extrait du critère R4 de la procédure d'inscription d'un élément sur la Liste Représentative du PCI de l'UNESCO.

51. HolLANDE François, « Mes trente engagements pour les Outre-mers », François Hollande 2012, p. 8 (georgespatient.fr/wp-content/uploads/2012/05/30-engagements-outre-mer.pdf, consulté le 7 juin 2019).

52. France-Antilles Guadeloupe du 14 novembre 2011, p. 2.

53. Ibid.

54. Wozan Monza, “Gwoka UNESCO” (piste 1), Maux Phrasés, 2016.

55. Gramsci Antonio, Cahiers de Prison, vol. 3 : Cahiers 10 à 13, Paris, Gallimard, 1983 [1948-1951], p. 394. 
56. Nom donné à l'élément sur la LR du PCI. C'est en effet ainsi que les membres du « Lyannaj Pou Gwoka » ont choisi d'identifier le Gwoka sur le formulaire officiel d'inscription sur la Liste Représentative du PCI.

57. AGEG, Rapport Culturel, p. 43.

58. GRAMSCI, Cahiers de prison.

59. DANIEL Justin, «Les formes d'expression politique dans la Caraïbe : ambivalence et paradoxe de la production de l'ordre politique ", BERTHOMIÈRE William et CHIVALLON Christine (dir.), Les diasporas dans le monde contemporain : un état des lieux, Paris / Pessac, Karthala /MSHA, 2006, p. 370. 60. воRтоLотTо Chiara (dir.), Le patrimoine culturel immatériel : enjeux d'une nouvelle catégorie, Paris, MSH, 2011.

61. GIVRE Olivier, «Savoirs et pouvoirs, stratégies et tactiques dans l'arène patrimoniale du Nestinartsvo (Bulgarie) ", Civilisations, vol. 61, n 1, 2012, p. 103.

62. PALUMBo Berardino, "L'UNESCO et le campanile: patrimoine universel et patrimoines locaux », FABRE Daniel et IUSO Anna (dir.), Les monuments sont habités, Paris, MSH, 2010, p. 157.

63. Ibid.

64. Ibid.

65. BORTOLOTTO Chiara et BERLINER David, «Introduction : le monde selon l'Unesco », Gradhiva, ${ }^{\circ}$ 18, 2013, p. 4-21; GIVRE, « Savoirs et pouvoirs, stratégies et tactiques dans l'arène patrimoniale du Nestinartsvo »; PALUMBO, «L'UNESCO et le campanile »; SANDRONI Carlos, « L'ethnomusicologue en médiateur du processus patrimonial: le cas de la samba de roda », воRтоцотто Chiara (dir.), Le patrimoine culturel immatériel : enjeux d'une nouvelle catégorie. p. 233-252.

66. PALUMBO, «L'UNESCO et le campanile ».

67. SANDRONI, «L'ethnomusicologue en médiateur du processus patrimonial ».

68. HотtiN, « L'ethnologie, un métier du patrimoine?».

\section{RÉSUMÉS}

Paris, le 26 novembre 2014 : le gwoka de Guadeloupe est inscrit sur la Liste Représentative (LR) du Patrimoine Culturel Immatériel (PCI) de l'humanité de l'UNESCO. Musique de tambour et de chant responsorial, le gwoka traditionnel se décline aujourd'hui en sept rythmes caractéristiques exprimés simultanément par des tambours appelés ka et des danseurs solistes. Il se joue la nuit, lors de soirées festives appelées léwoz. Cet article souhaite contribuer à la réflexion sur les logiques d'appropriation $\mathrm{du}$ concept de patrimoine culturel. Fondé sur des données ethnographiques recueillies entre 2006 et 2015 dans le cadre d'une observation participante, il examine l'impact du processus d'inscription du gwoka sur la LR du PCI de l'UNESCO et met en lumière les enjeux politiques de cette appropriation pour les acteurs du gwoka. Cet article commence par une présentation du Rapport culturel publié par l'Association Générale des Étudiants Guadeloupéens (AGEG) en 1970. Ce Rapport culturel est un texte fondateur qui développe « une revendication spécifique privilégiant le gwoka comme élément central de la personnalité culturelle populaire guadeloupéenne ». Ce document est à l'origine des premiers efforts de mise en patrimoine du gwoka par les Guadeloupéens eux-mêmes dans les années 1970 et 1980 . Nous nous intéressons ensuite à la mise en circulation de la notion de PCI en Guadeloupe, où la réflexion autour de la patrimonialisation est déjà bien avancée et où des actions de mise en patrimoine sont déjà en cours. Contrairement à ce qui a pu être observé ailleurs, cette mise en 
circulation a pour particularité d'avoir été initiée par les porteurs de tradition et non par l'État. Enfin nous nous arrêterons sur les débats passionnés que le projet d'inscription a suscités, transformant le gwoka en « arène patrimoniale ».

Paris, November 26, 2014: Guadeloupe gwoka is inscribed on the Representative List (RL) of the UNESCO Convention for safeguarding Intangible Cultural Heritage (ICH). Traditional gwoka is a dance performed to the sound of ka drums and call-and-response singing. It takes place at night during festive events called léwoz. In this article, we want to contribute to the discussion of the ways in which communities make use of the notion of Cultural Heritage. It is based on ethnographic data gathered between 2006 and 2015 through participant observation. It discusses the impact that gwoka's inscription on the RL of the ICH had on the gwoka community. It wants to shed light on some of the political stakes that inscribing a cultural expression on the RL of the ICH Convention entails. This article opens on a presentation of a brochure titled Rapport culturel, which was published in 1970 by the General Association of Guadeloupean Students (AGEG). The Rapport culturel is a founding document that formulates a "specific claim that gwoka is a core component of the cultural personality of the Guadeloupean people". This document compelled Guadeloupeans of the 1970s and 80s to undertake a variety of actions aiming at making Gwoka a defining element of their cultural heritage. Second, we examine the ways in which the notion of ICH began to circulate in Guadeloupe, a place where heritagisation processes are well underway. Contrary to what has been observed elsewhere, in Guadeloupe the tradition bearers were the ones who initiated the spread of $\mathrm{ICH}$, not the State. Finally, we focus on the vibrant debate the inscription process has sparked, thus transforming gwoka into a political arena where notions of power and identity are contested, re-discussed and re-imagined.

\section{INDEX}

Keywords : intangible cultural heritage, Guadeloupe, gwoka, UNESCO, AGEG

Mots-clés : patrimoine culturel immatériel, Guadeloupe, gwoka, UNESCO, AGEG

\section{AUTEURS}

\section{FLORABELLE SPIELMANN}

ATER à l'Université Paris 8 Vincennes-Saint-Denis, Florabelle Spielmann est anthropologue, spécialiste des musiques et sociétés de la Caraïbe. Elle a travaillé à la Maison des Cultures du Monde à Paris entre mai 2007 et novembre 2009 sous la direction de Chérif Khaznadar puis d'Arwad Esber. Entre 2010 et 2014, elle a participé aux réunions de travail du comité « Lyannaj pou Gwoka ", groupe d'acteurs du gwoka qui a conduit le projet d'inscription en Guadeloupe. Elle a également travaillé à l'élaboration du dossier de candidature du gwoka à la Liste représentative du PCI.

\section{DOMINIQUE CYRILLE}

Dominique Cyrille, docteure en Musicologie, s'est spécialisée dans l'étude des expressions culturelles de la Caraïbe. Elle a été Assistant Professor of African and African-American Studies à Lehman College CUNY de 1999 à 2008 et consultante auprès de la médiathèque Bettino Lara de Basse-Terre et du Festival de Gwoka pour l'organisation du Séminaire d'Ethnomusicologie Caribéenne de 2002 à 2008. De septembre 2008 à novembre 2015 elle a été responsable de la mission Patrimoine de Rèpriz, centre des musiques et danse traditionnelles et populaires de 
Guadeloupe. À ce titre, elle a participé à la création et à la mise en place du «Lyannaj pou Gwoka » et a pris une part active à l'élaboration du dossier d'inscription du gwoka sur la Liste représentative de la convention de l'UNESCO. 